\title{
Translin and Trax differentially regulate telomere-associated transcript homeostasis
}

\author{
Natalia Gomez-Escobar ${ }^{1, *}$, Nasser Almobadel ${ }^{1, *}$, Othman Alzahrani ${ }^{1, *}$, Julia \\ Feichtinger ${ }^{2,3, *}$, Vicente Planells-Palop ${ }^{1, *}$, Zafer Alshehri $^{1}$, Gerhard G. Thallinger ${ }^{2,3}$, \\ Jane A. Wakeman ${ }^{1}$ and Ramsay J. McFarlane ${ }^{1}$ \\ ${ }^{1}$ North West Cancer Research Institute, School of Medical Sciences, Bangor University, Bangor, Gwynedd, United Kingdom \\ ${ }^{2}$ Computational Biotechnology and Bioinformatics Group, Institute of Molecular Biotechnology, Graz University of Technology, \\ Graz, Austria \\ ${ }^{3}$ Omics Center Graz, BioTechMed Graz, Graz, Austria \\ * These authors have contributed equally to this work
}

Correspondence to: Ramsay J. McFarlane, email: r.macfarlane@bangor.ac.uk

Keywords: telomeres, Translin, Trax, C3PO, TERRA, Chromosome Section

Received: February 15, $2016 \quad$ Accepted: April 19, 2016

Published: May 10, 2016

\section{ABSTRACT}

Translin and Trax proteins are highly conserved nucleic acid binding proteins that have been implicated in RNA regulation in a range of biological processes including tRNA processing, RNA interference, microRNA degradation during oncogenesis, spermatogenesis and neuronal regulation. Here, we explore the function of this paralogue pair of proteins in the fission yeast. Using transcript analysis we demonstrate a reciprocal mechanism for control of telomere-associated transcripts. Mutation of $t f \times 1^{+}$(Trax) elevates transcript levels from silenced sub-telomeric regions of the genome, but not other silenced regions, such as the peri-centromeric heterochromatin. In the case of some sub-telomeric transcripts, but not all, this elevation is dependent on the Trax paralogue, Tsn1 (Translin). In a reciprocal fashion, Tsn1 (Translin) serves to repress levels of transcripts (TERRAs) from the telomeric repeats, whereas Tfx1 serves to maintain these elevated levels. This reveals a novel mechanism for the regulation of telomeric transcripts. We extend this to demonstrate that human Translin and Trax also control telomere-associated transcript levels in human cells in a telomere-specific fashion.

\section{INTRODUCTION}

The faithful segregation of chromosomes during the eukaryotic cell cycle is a multifaceted event, failures in which can drive diverse human diseases, including cancers [1]. Protection of the termini of linear eukaryotic chromosomes, the telomeres, during this process is essential to prevent nucleolytic chromosomal degradation [2]. Telomere maintenance is normally mediated by a specific DNA polymerase termed telomerase, which is capable of extending telomeric DNA independently of the major genomic replisome [3, 4]. Telomere length control provides a molecular limiter on cellular proliferative potential as telomerase activity is repressed in most healthy somatic cells. Without length homeostasis being maintained, telomeres will shorten with each division, ultimately resulting in cellular senescence $[3,5]$.
Proliferating cancer cells must override this senescence pathway, either via the activation of telomerase, chromosomal circularization or activation of alternative telomere maintenance pathways [6]. Telomeres also contribute to the correct chromosomal architecture within the nucleus [7] and telomere dysfunction has been associated with a range of human genetic diseases [8].

Telomeric DNA is highly repetitive in nature and associates with a plethora of telomere regulatory proteins to form a unique nucleoprotein structure $[2,3,9]$. Chromosomal regions adjacent to the telomeres are often subjected to heterochromatic transcriptional silencing $[4,10,11]$ although long noncoding telomeric repeatcontaining RNA (TERRA) is transcribed from telomeric DNA repeats [12-16]. The function(s) and regulators of TERRAs remains poorly defined, but TERRAs have been implicated in a range of telomere-associated processes, 
including DNA damage response [12-16].

The human protein Translin was first identified by virtue of its ability to bind to the breakpoint junctions of chromosomal translocations in lymphoid malignancies [17] and other genetic diseases [18]. It was independently identified in the mouse as the testis-brain RNA binding protein [19]. Translin is highly conserved in eukaryotes and archaea and has a paralogue binding partner Trax (Translin-associated protein X) [18]. The Translin-Trax complex has the ability to bind to nucleic acid, with a bias towards RNA, and it possesses RNA nucleolytic activity [18, 20-23]. DNA sequence binding preferences has led to the suggestion that Translin may function in telomere regulation, although to date there is no direct evidence for this [24]. Telomeres directly interact with proteins associated with homologous recombination and DNA double-strand break (DSB) repair [25], and Trax has recently been demonstrated to have a role in the murine DNA damage response via association with the ATM pathway [26]. Whilst a role for Translin in this response has not yet been found, Translin function has also been linked to the mammalian DNA damage response [27].

Since their initial identification, Translin and Trax have been implicated in a wide range of nucleic acid processing pathways, including tRNA maturation and mRNA regulation both in spermatogenesis and neuronal dynamics, with mutations in the Translin binding site of $B D N F$ (brain-derived neurotrophic factor) mRNA resulting in memory deficits and psychiatric disorders $[18,28,29]$. Recently, Drosophila and mammalian Translin and Trax were revealed to be the constituents of the C3PO complex [22, 30-32], which is responsible for assisting the removal of the passenger RNA strand from small interfering RNAs involved in Argonaute-dependent RNAinduced transcriptional silencing [33]. In most activities studied to date, Translin and Trax have been shown to function in unison, and the maintenance of Trax stability is a conserved function of Translin [18, 27]. Importantly, a direct role in oncogenesis has recently been reported for Translin and Trax in cancers that are haploinsufficient for Dicer, as they degrade pre-microRNAs that would be processed to microRNAs by a full Dicer complement to maintain tumour suppression [34]. This has led to the suggestion that Translin and Trax could provide drug targets in Dicer haploinsufficient tumours [34].

Here we reveal previously unknown roles for Translin and Trax in controlling homeostasis of distinct telomere-associated transcripts in both fission yeast and human cells. Moreover, we demonstrate that Translin and Trax can act independently, indicating that they do not function solely as a heteromeric complex, but have interrelated roles in controlling telomere-associated transcript.

\section{RESULTS}

\section{Trax, but not Translin, represses sub-telomeric transcript levels}

The extensive conservation of Translin and Trax indicate they serve a fundamentally important biological role. Despite this, deletion of both the Translin $\left(t s n 1^{+}\right)$and $\operatorname{Trax}\left(t f x 1^{+}\right)$genes of the fission yeast Schizosaccharomyces pombe does not result in any readily detectable phenotypic change $[35,36]$. Given the finding that Translin and Trax regulate RNA dynamics in other organisms, we set out to determine whether S. pombe Tsn1 and $\mathrm{Tfx} 1$ are involved in transcript regulation. To do this, we used tiling arrays to analyse the transcriptome and to identify any changes when $t s n 1^{+}$and $t f x 1^{+}$are mutated. Comparison of the wild-type and tsn $1 \Delta$ transcripts reveals no statistically meaningful difference. However, comparison of the wild-type and tf $x 1 \Delta$ strains revealed one significant change: an up-regulation of sub-telomeric th gene transcripts in the $t f x 1 \Delta$ mutant (Figure 1). It should be noted that the annotated DNA sequences for $t$ th 1 and th 2 genes are $100 \%$ identical so we cannot rule out a th 1 or tlh2-only dysregulation (i.e. a specific sub-telomeric region). The double $t s n 1 \Delta t f x 1 \Delta$ mutant exhibits the same th transcript elevation seen in the $t f x l \Delta$ single mutant (Figure 1). Not only is the elevation of the $t$ lh transcripts the first measurable phenotype of a $t f x 1 \Delta$ mutant, it also reveals a functional separation between $\mathrm{Tfx} 1$ and Tsn 1 in S. pombe. S. pombe Tsn1 functions to stabilize Tfx1 [35] and in the absence of Tsn1 there are considerably reduced levels of Tfx1 [35]. The th transcript data indicate that the low levels of Tfx 1 in the tsn $1 \Delta$ background remain sufficient to maintain th transcript repression.

There are four th paralogues within the S. pombe genome (although only two are currently annotated and have their transcription measured using the tiling arrays; C. Norbury, personal communication). One paralogue is located within the sub-telomeric regions of each of the four telomeres for $S$. pombe chromosomes 1 and 2 ( $S$. pombe has three chromosomes and the sub-telomeric regions of chromosome 3 are unique as they consist of rDNA repeats). The $t l h$ genes are $B L M$ gene orthologues of unknown function, although they have been implicated in recovery from telomerase loss crisis and they are normally subjected to transcriptional silencing [37]. Given the fact that sub-telomeric regions are governed by RNA interference-mediated transcriptional silencing, which also regulates the heterochromatic transcriptional silencing in the outer repeat regions of the three S. pombe centromeres [10], we carefully examined transcription from these centromeric repeat regions in the $t s n 1 \Delta$ and $t f x 1 \Delta$ mutants. No measurable change in transcript levels between mutants and wild-type was measured for either 
DNA strand (Figure 2, Figure S1-S2). Collectively, these data indicate that Tfx1, but not Tsn1 functions in subtelomeric, but not centromeric, transcript regulation.

\section{Loss of Tfx1 suppresses the requirement for Ago1}

Similar to the loss of Tfx1, the loss of the telomere regulator Taz1 [38] has also been demonstrated to elevate the $t$ th transcript levels [39]. Moreover, loss of Taz1 function has been shown to partially suppress sensitivity to the microtubule destabilizing drug thiabendazole (TBZ) of cells mutated in the RNA interference regulator gene agol $^{+}$[40] (Figure S3). It has been postulated that this suppression occurs due to heterochromatin factors mediating the sub-telomeric silencing being 'released' from the sub-telomeric regions to relocate and enhance the centromeric function that is compromised due to loss of Ago1 [40]. To determine whether loss of Tfx1 results in a similar phenotypic outcome as loss of the known telomere regulator Taz1, we generated ago $1 \Delta t f x 1 \Delta$ and agol $\Delta$ tsn $1 \Delta$ double mutants and tested their response to TBZ. Consistent with the $t$ th transcript up-regulation data (Figure 1), Figure 3A shows that loss of Tfx1, but not Tsn1 partially suppresses the need for Ago1, which is the same for the suppression reported upon loss of Tazl [40] (Figure S3), consistent with a functional link for Tfx1 to telomeric regulation.

\section{Tfx1 and Tsn 1 do not regulate telomere length}

Taz1 is required to limit telomere length and in the absence of Taz1 telomeres become highly elongated [38]. Given the phenotypic similarities between tazl $\Delta$ and tf $x 1 \Delta$ mutants, we wished to determine whether Tfx 1 also contributed to telomere length regulation. To address this, Southern blot analysis was carried out to compare telomere
A

tlh1
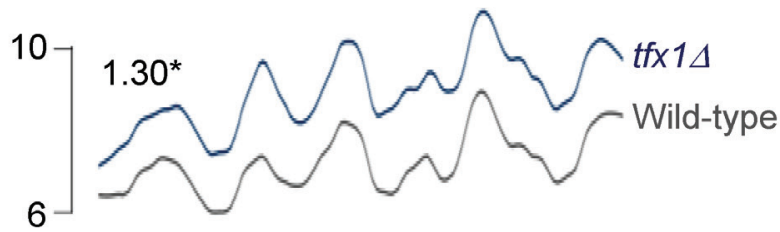

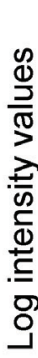

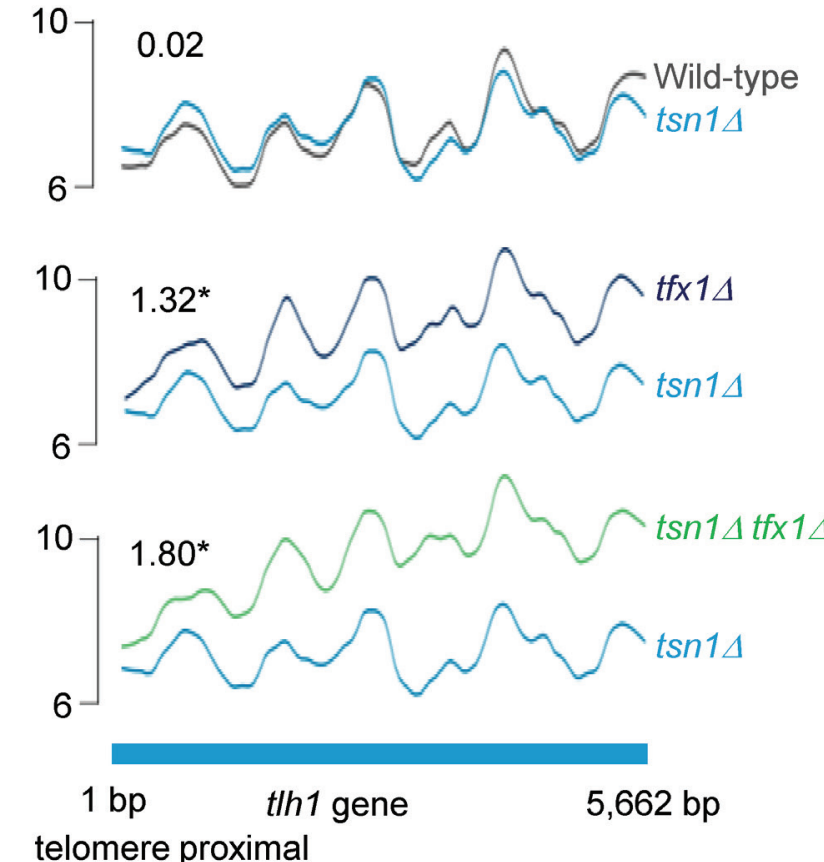

B

tlh2

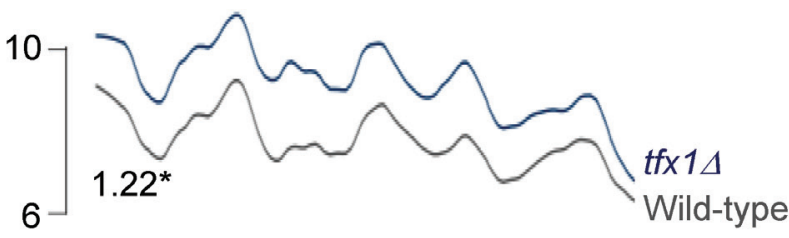

10
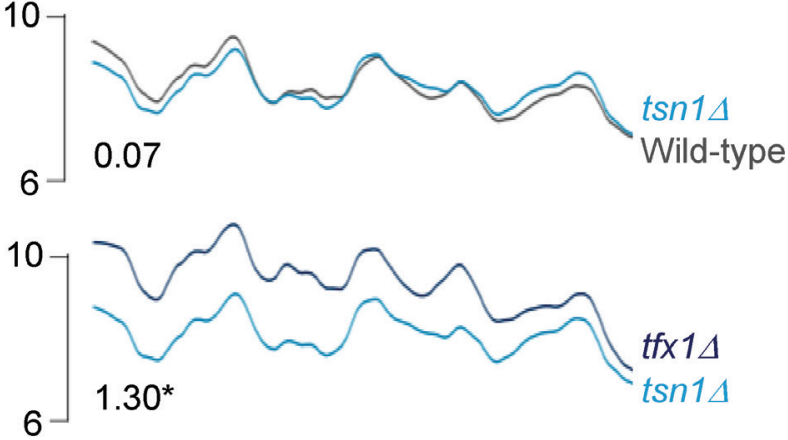

6

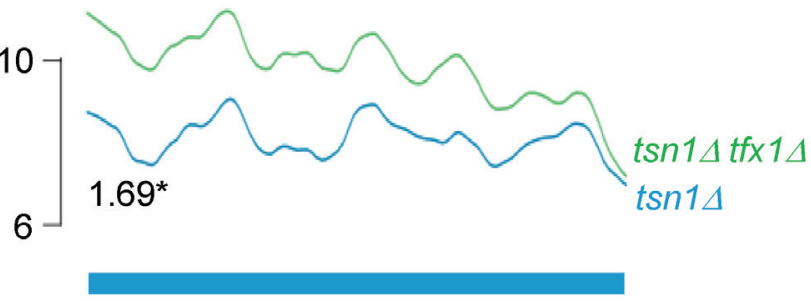

$4,526,885 \mathrm{bp}$ th2 gene telomere proximal

Figure 1: Tfx1 (Trax), but not Tsn1 (Translin) regulates sub-telomeric transcript levels in $S$. pombe. Both images show relative transcript levels obtained from tiling microarrays. A. $t$ th 1 gene transcripts are elevated in a $t f x 1 \Delta$ mutant, but not in $t s n 1 \Delta$ mutant. Elevation is maintained in a $t f x 1 \Delta t s n 1 \Delta$ double mutant. Base pair (bp) designation represents the nucleotide annotation position for $S$. pombe chromosome 1 (thl ORF is on the reverse strand, hence reverse strand profile is shown). B. A similar profile is seen for the other annotated sub-telomeric $t$ lh gene, $t$ lh 2 . Fold change values are given within the plots $(*=\mathrm{P}<0.05)$. 
lengths between a $\operatorname{taz} 1 \Delta, \operatorname{tsn} 1 \Delta$ and $t f x 1 \Delta$ mutants (and the $t f x 1 \Delta t \operatorname{sn} 1 \Delta$ double mutant). $t \operatorname{sn} 1 \Delta$ and $t f x 1 \Delta$ single mutants had mean telomere lengths indistinguishable from the wild-type, whereas telomeres in the tazl $\Delta$ mutant were greatly elongated (Figure $3 \mathrm{~B} / \mathrm{C}$ ). These data indicate that the de-silencing of the $t$ lh genes seen in the $t f x 1 \Delta$ mutant is not related to telomere length dysregulation, as it is in the $\operatorname{taz} 1 \Delta$ mutant.

\section{Tsn1 and Tfx1 differentially regulate telomeric transcripts}

TERRA production from telomeric DNA template is a conserved feature of telomeres in both humans and fission yeast [12-16]. Other sub-telomeric transcripts, termed ARRETs have also been detected in $S$. pombe for the sub-telomeric regions on chromosomes 1, 2 and occasionally 3 [41, 42]. ARRET and TERRA sequences are not covered on the tiling arrays used in this study.

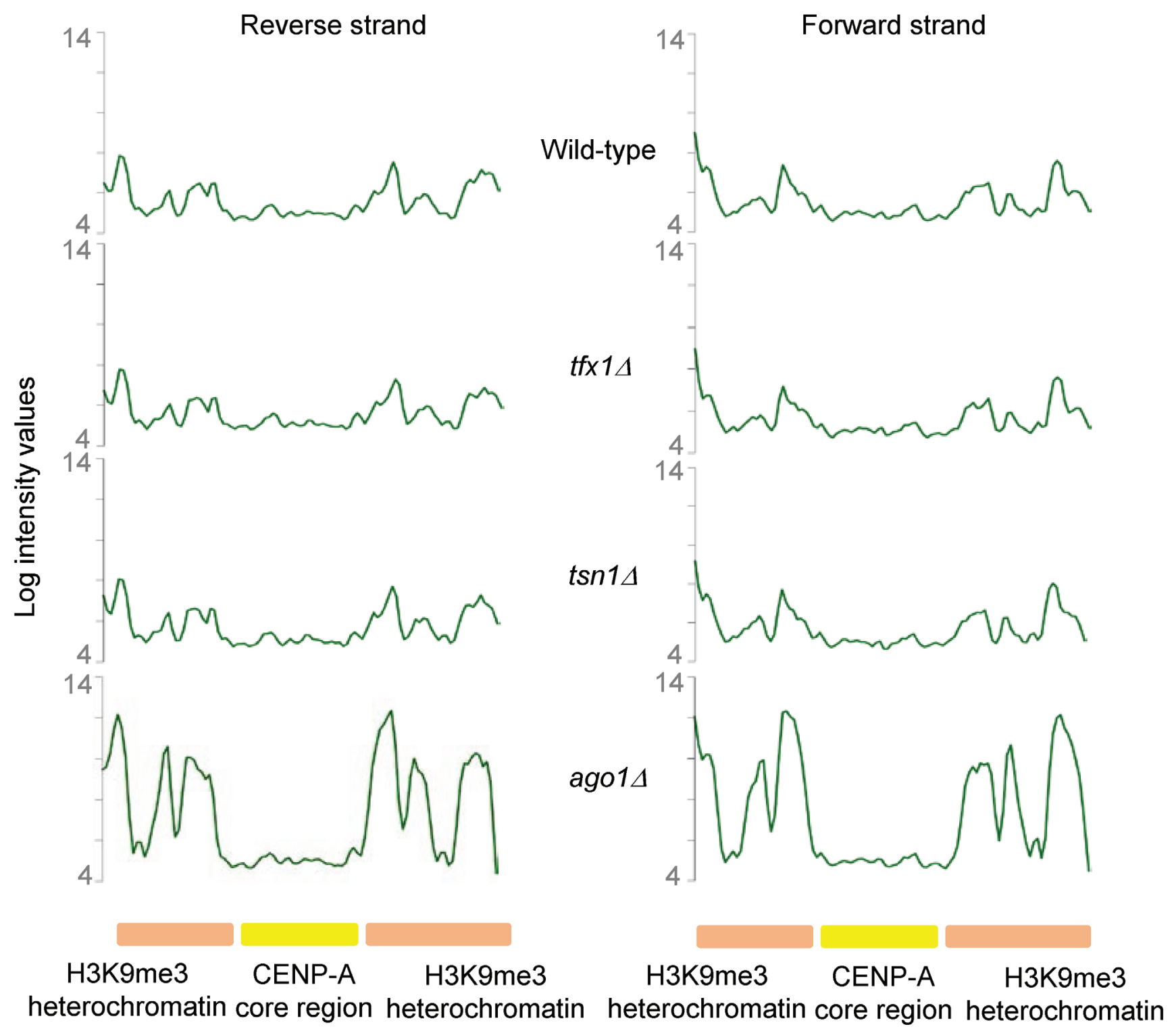

Figure 2: Centromeric transcript levels remain unaltered upon loss of Tsn1 or Tfx1. Centromeric transcript levels remains unaltered in either $t f x 1 \Delta$ or $t s n 1 \Delta$ mutants (middle two profiles) compared to the wild-type (top profile). The profile for an $a g o l \Delta$ mutant is given as a control for a desilencing mutant (bottom profile). The approximate spread of heterochromatic and centromere core regions are given (bottom line). The profiles are for the forward (right) and reverse (left) strand of cen 1 . The $S$. pombe nucleotide coordinates shown for cen 1 are chromosome 1: 3,754,000 - 3,790,000. Both strands for cen 2 and cen 3 show similar inactivation in $t f x 1 \Delta$ and $t s n 1 \Delta$ mutants and are given in Figure S1 and S2 respectively. 
To further confirm the regulation of sub-telomeric transcripts by Tfx 1 , we examined ARRET (immediately sub-telomeric regions) levels in $t f x 1 \Delta$ and $t s n 1 \Delta$ strains using RT-PCR/RT-qPCR [43]. Loss of Taz1 gives elevated ARRET levels, consistent with activation of $t$ th genes in the absence of Taz1 [39, 42]. Loss of Tfx1 resulted in an approximately two-fold elevation in ARRETs, again, consistent with the $t$ th transcripts (Figure 4). However, unlike the $t h$ transcript elevation, additional mutation of ts $n 1^{+}$results in a reduction of ARRET transcripts back to wild-type levels, indicating an interplay between Tfx1 and Tsn1 for ARRET regulation, but not $t$ lh transcript regulation (Figure 1, Figure 6); so, in the absence of Tfx1, Tsn 1 is required to maintain elevated ARRET levels.

Taz1-defficient cells also exhibit elevated levels of TERRAs [42]. Telomeric DNA is not extended in the tf $x 1 \Delta$ mutant (Figure $3 \mathrm{~B} / \mathrm{C}$ ) and so we wished to address whether the regulation of telomere-associated transcripts extended into the TERRA encoding telomeric regions. To do this, we analysed TERRA levels in the various mutants. Using RT-PCR/RT-qPCR we could only detect very limited TERRA levels in the wild-type (Figure 4). However, both $\operatorname{taz} 1 \Delta$ and $t \operatorname{sn} 1 \Delta$ mutants exhibited clearly elevated levels of TERRA (Figure 4), whereas levels in the tf $x 1 \Delta$ mutant were indistinguishable from the wild-type. This surprise finding indicates that Tsn1 is required for suppression of TERRA levels whereas Tfx 1 is required for suppression of sub-telomeric transcripts ( $t$ lh genes and ARRETs). Interestingly, additional mutation of $t f x 1^{+}$in the $t s n 1 \Delta$ background results in restoration of TERRA levels to those of the wild-type indicating that in the absence of Tsn1, Tfx1 stabilizes the elevated levels of TERRA (Figure 4). So, importantly, these findings indicate an inverted reciprocal relationship between Tfx 1 and Tsn 1 for sub-telomeric and telomeric transcript regulation, respectively. Upon loss of Tfx1, ARRETs, but not TERRAs, are elevated in a Tsn1-dependent fashion, and upon loss of Tsn1, TERRAs, but not ARRETs, are elevated in a Tfx1-dependent fashion. The requirement for Tsn 1 to maintain elevated sub-telomeric transcripts only applied to the ARRETs and not the $t$ lh genes indicating a positional constraint on this relationship, possibly governed by proximity to the telomeric DNA where Tsn 1 functions to suppress TERRA transcript levels. $S$. pombe also produces other telomeric and sub-telomeric transcripts (termed ARIAs and $\alpha$-ARRETs respectively) $[41,42]$, but we could not distinguish RT-PCR products of these transcripts from transcripts produced by first strand cDNA primed by endogenous priming; for both TERRA and ARRET analysis lack of first strand primers produced no PCR products and so problematic endogenous priming was eliminated (Figure 4).

\section{Translin and Trax regulate human TERRA levels}

TERRA was originally detected in mammals [44, 45]. To explore whether the role of Translin in suppressing TERRA levels was conserved, we measured TERRAs for the $\mathrm{q}$ arm of chromosome 10 and the sex chromosomes $(\mathrm{X} / \mathrm{Y})[46]$ in human SW480 cells following siRNA depletion of either TSN1 (Translin) or TSNAX (Trax) expression (Figure S4). Figure 5 shows that TERRA levels were elevated in TSN-depleted cells, but not in TSNAXdepleted cells for the 10q telomere. The TSNAX-depleted cells showed reduced TERRA levels for all telomeres tested.

These findings indicate that the separation of roles for Translin and Trax in TERRA regulation is conserved from $S$. pombe to some telomeres in humans. The $10 \mathrm{q}$ TERRA data indicate that Translin serves to suppress levels of some human TERRAs, whereas Trax serves to stabilize/maintain their levels, raising the possibility of a reciprocal TERRA stabilization mechanism at play. The same relationship was not readily observed for the q arm of the sex chromosomes, where depletion of TSN did not result in elevated X/Yq TERRAs, but depletion of TSNAX reduced TERRAs (Figure 5). These data indicate a more complex picture in human cells, with the potential for a telomere-specific requirement for distinct TSN and TSNAX functions.

\section{DISCUSSION}

\section{Translin and Trax function to regulate telomere transcripts}

Translin and Trax influence distinct biological processes in a species- and tissue-specific manner [18]. The majority of the biological roles appear to require the modulation of RNA (not DNA) molecules, including mRNAs, tRNA precursors, microRNAs and siRNA passenger strands [18], although the functional significance of chromosomal translocation breakpoint junction binding capability remains unclear [17]. Here we demonstrate that Translin and Trax control telomere-associated transcript abundance, but do so in distinct fashions. That these factors function in such a fundamental process as telomere transcript regulation, yet their disruption does not result in significant perturbation of proliferative potential, in fission yeast at least, indicates that there is a degree of tolerance for alteration of telomeric transcript levels. In mammalian cells loss of Translin and Trax function has been linked to reduced cell proliferation [47-49], but this may relate to disruption of non-telomeric functions in these cells. Given that dysregulation of telomeric transcripts is the only defect in $t s n 1 \Delta$ and $t f x 1 \Delta$ mutants of $S$. pombe, this makes 
it an excellent system in which to study this important function of these proteins in the absence of perturbation of other biological roles.

TERRAs control a range of telomere-associated functions, including regulation of telomerase activity, telomere length control, telomeric heterochromatinization and cellular differentiation [12-16]. TERRA has both positive and negative influences upon telomere length regulation, with cell cycle and tissue-specific influences [16]. In budding yeast, loss of Rat1 ribonuclease processing results in accumulation of TERRAs and inhibition of telomerase leading to telomere shortening [50]. Whilst we show here that loss of tsn 1 also results in TERRA accumulation, this alone does not appear to be sufficient for measurable telomere length alteration or reduction in cell viability. This function of tsn 1 seems

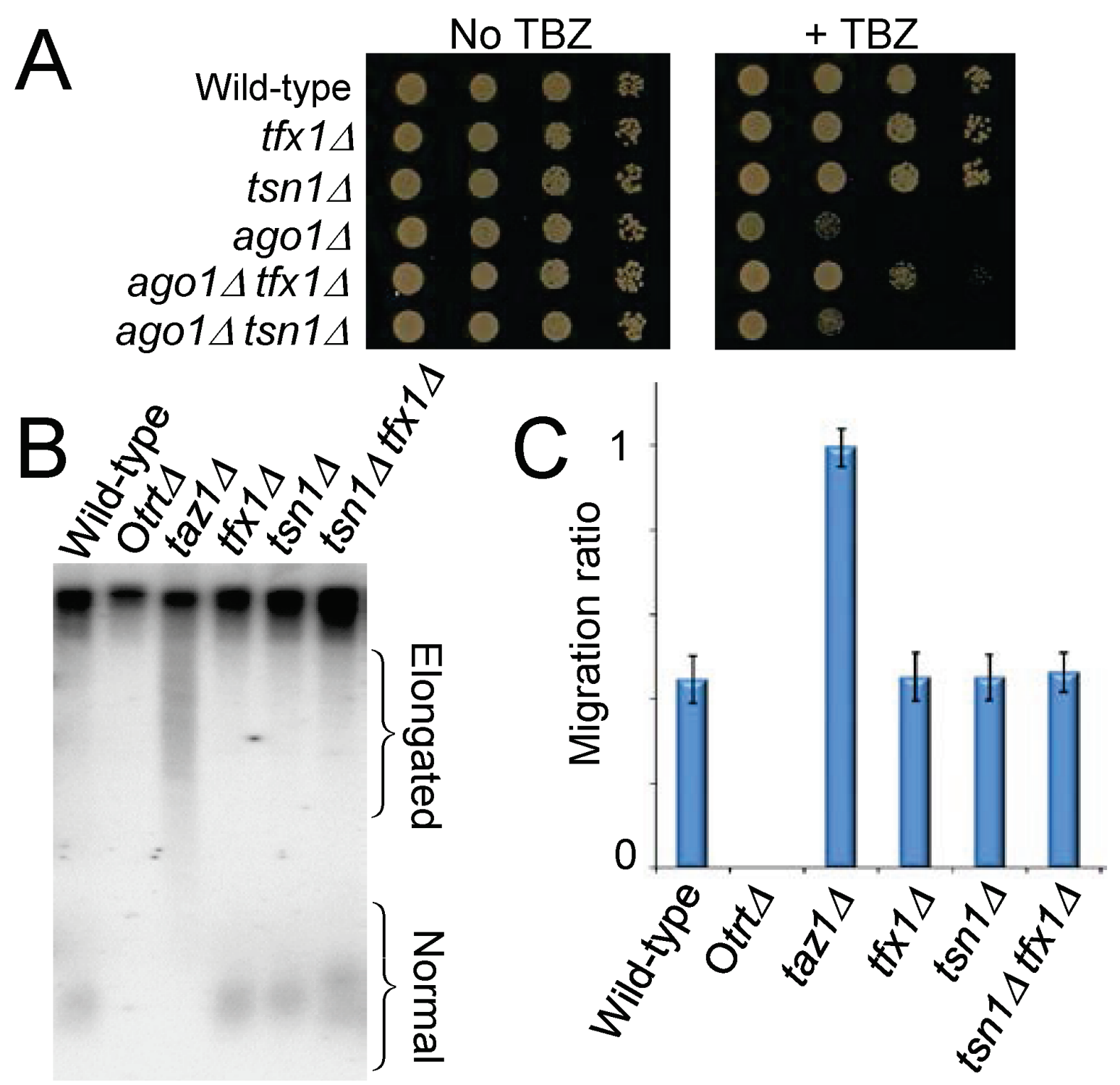

Figure 3: Loss of Tfx1 results in a telomere-defective phenotype, but telomere length is unaltered. A. Suppression of TBZ sensitivity of an agol $\Delta$ mutant is a feature of telomere regulator proteins [38]. The tf $x 1 \Delta$ mutant, like the taz1 $\Delta$ mutant [38] (Figure S3), partially suppresses the $a g o 1 \Delta \mathrm{TBZ}$ sensitivity, whereas a $t s n 1 \Delta$ mutation does not. B. Southern blot probed with a telomere-specific probe showing that both $t f x 1 \Delta$ and $t s n 1 \Delta$ mutants have telomeres with lengths similar to the wild-type. The tazl $1 \Delta$ mutant had highly elongated telomeres. The Otrt $\Delta$ strain has circular chromosomes without telomeres and serves as a control for the probe specificity. C. Quantification of telomere fragment migration ratios demonstrates no statistically significant difference between the mean telomere lengths of the $t f x 1 \Delta$, $t \operatorname{sn} 1 \Delta$ and wild-type strains. Mean migration values for at least four repeats were obtained. Pairwise Student's $t$-tests were conducted between all telomere containing strains (i.e. not the Otrt strain) and the wild-type. All $P$ values were $>0.05$ with the exception of the wildtype $v$ s. $\operatorname{taz} 1 \Delta$ analyses which was $<0.01$. Error bars are standard deviations. 
to be conserved, as depletion of human TSN results in elevation of TERRA from the $\mathrm{q}$ arm telomere of chromosome 10, although this same effect was not seen for the $\mathrm{q}$ arm of $\mathrm{X} / \mathrm{Y}$, which might reflect a requirement for distinct levels of TSN (siRNA does not cause full cellular depletion) or a telomere specificity for TSN function. Interestingly, a recent systematic approach to identify telomere DNA and TERRA interacting proteins did not identify TSN or TSNAX. This suggests that telomereassociated transcriptome regulation by TSN and/or TSNAX in humans is likely mediated by an indirect and/ or transient/labile association [51]. In S. pombe, Translin
(Tsn1) only forms weak interactions with other non-Trax (Tfx1) proteins [52], and possibly other macromolecules, and no interactions have been demonstrated with known telomere regulators.

\section{Distinct and joint functions for Translin and Trax}

Almost all studies to date, revealing a biological role for Translin and Trax, have demonstrated a close functional association between the two [18]. Here, we show both a joint and separate functional association between these paralogues. Firstly, the transcript up-regulation for sub-

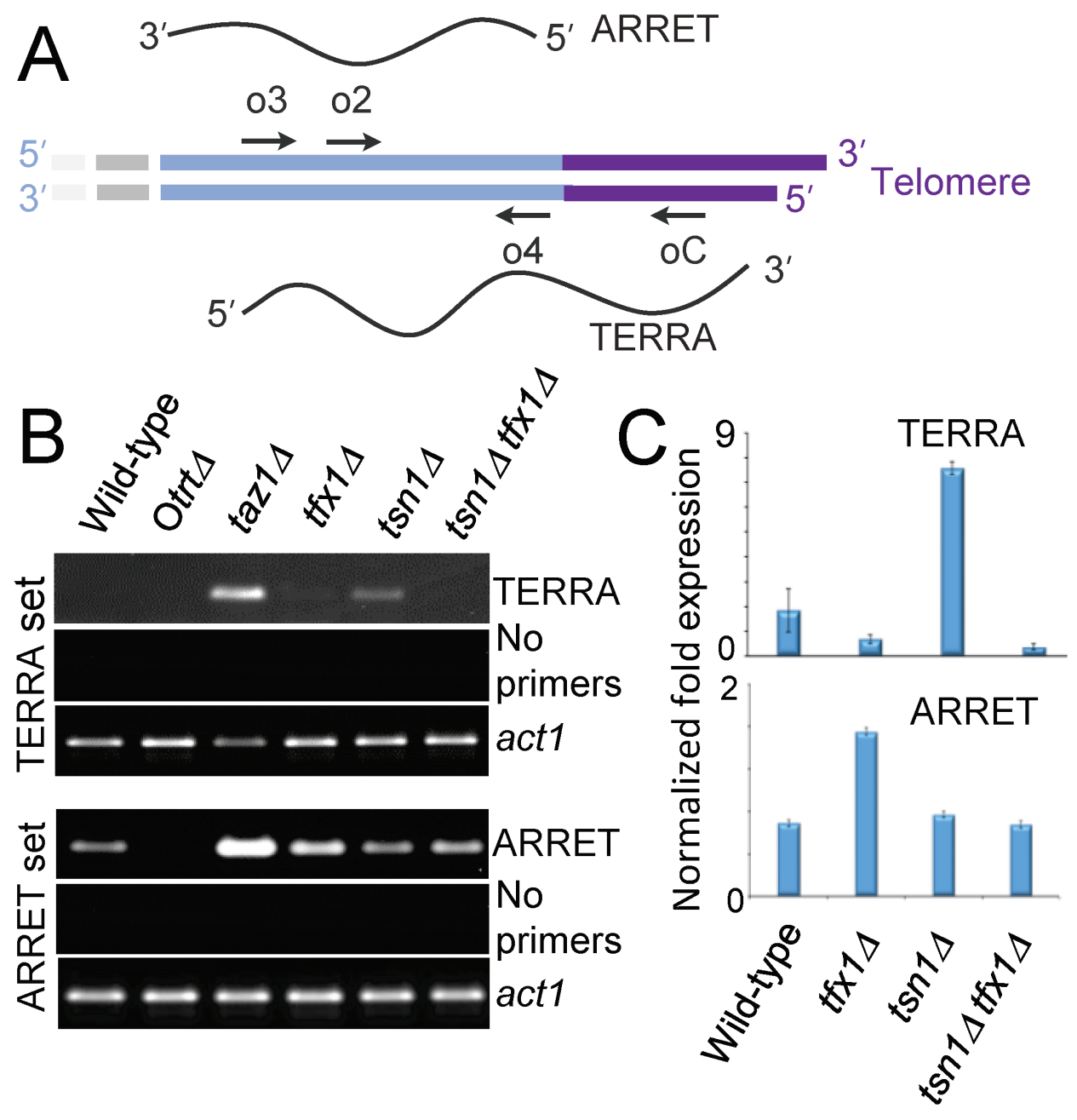

Figure 4: Translin and Trax regulate telomere-associated transcripts in fission yeast cells. A. A schematic of an S. pombe telomere showing the telomere (purple) and the sub-telomeric region (light blue; previously referred to as STE) [42]. The ARRET and TERRA transcripts are aligned to their approximate template location. Arrows indicate primer positions used for first strand cDNA synthesis and RT-PCR (for TERRAs cDNA priming used oC, PCR was primed using o2/o4; for ARRETs cDNA priming used o3 and PCR was primed using o2/o4; primer sequences and designations are derived from Greenwood and Cooper [42]). B. Agarose gels showing analytical RTPCR for TERRAs (first strand oC) and ARRETs (first strand o3). No primer controls indicate no first strand cDNA primers, demonstrating that PCR products are not due to endogenous first strand priming. RT-PCT for the $a c t 1^{+}$gene transcripts are given as a positive control. C. RT-qPCR for TERRAs and ARRETs in $t s n 1 \Delta$ and $t f x 1 \Delta$ cells. Error bars represent standard deviation. Student's $t$-test pairwise comparison were carried out for mean values between the wild-type $v s$. mutants. In all cases $P$ values were $>0.05$ with the exceptions of wild-type $v s$. $t s n 1 \Delta$ for TERRA levels (upper set) and wild-type $v s$. $t$ f $1 \Delta$ for ARRET levels (lower set) where $P$ values were $<0.01$. 
telomeric regions only occurs when $t f x 1^{+}$is mutated but not upon $t s n 1^{+}$mutation. The elevation of ARRET levels is $t s n 1$-dependent (Figure 4), but the $t$ th transcripts remain elevated in the $t \operatorname{sn} 1 \Delta t f x 1 \Delta$ double mutant (Figure 1), indicating there may be a transcript/positional specificity to this regulation. Conversely, the elevation of TERRA levels in the $t s n 1 \Delta$ mutant is $t f x 1$-dependent. These findings indicate that there is a reciprocal control mechanism operating between Tsn 1 and Tfx 1 to maintain telomere-associated transcriptional homeostasis (Figure

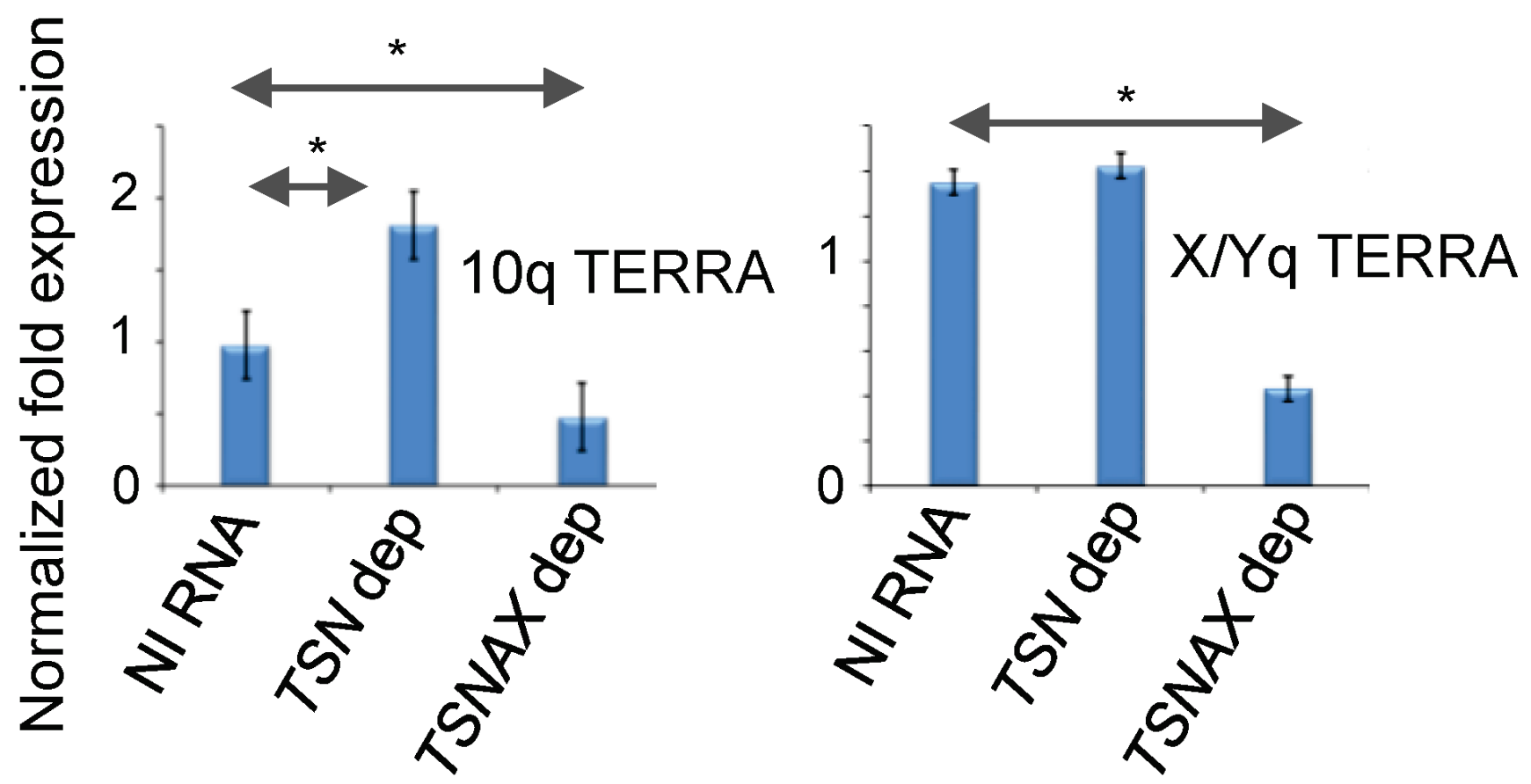

Figure 5: Human Translin and Trax influence TERRA levels differentially for distinct telomeres. RT-qPCR for human TERRAs for the telomeres of the $\mathrm{q}$ arm of chromosome 10 (left) and the $\mathrm{q}$ arm of the sex chromosome (X/Y; right) in TSN and TSNAX depleted SW480 cells. NI RNA = non-interfering RNA. Error bars represent standard deviations. Student's $t$-test pairwise comparison were carried out for mean values between NI RNA treated cells and siRNA depleted cells. Only comparisons marked with * showed statistical significance $(P<0.05)$.

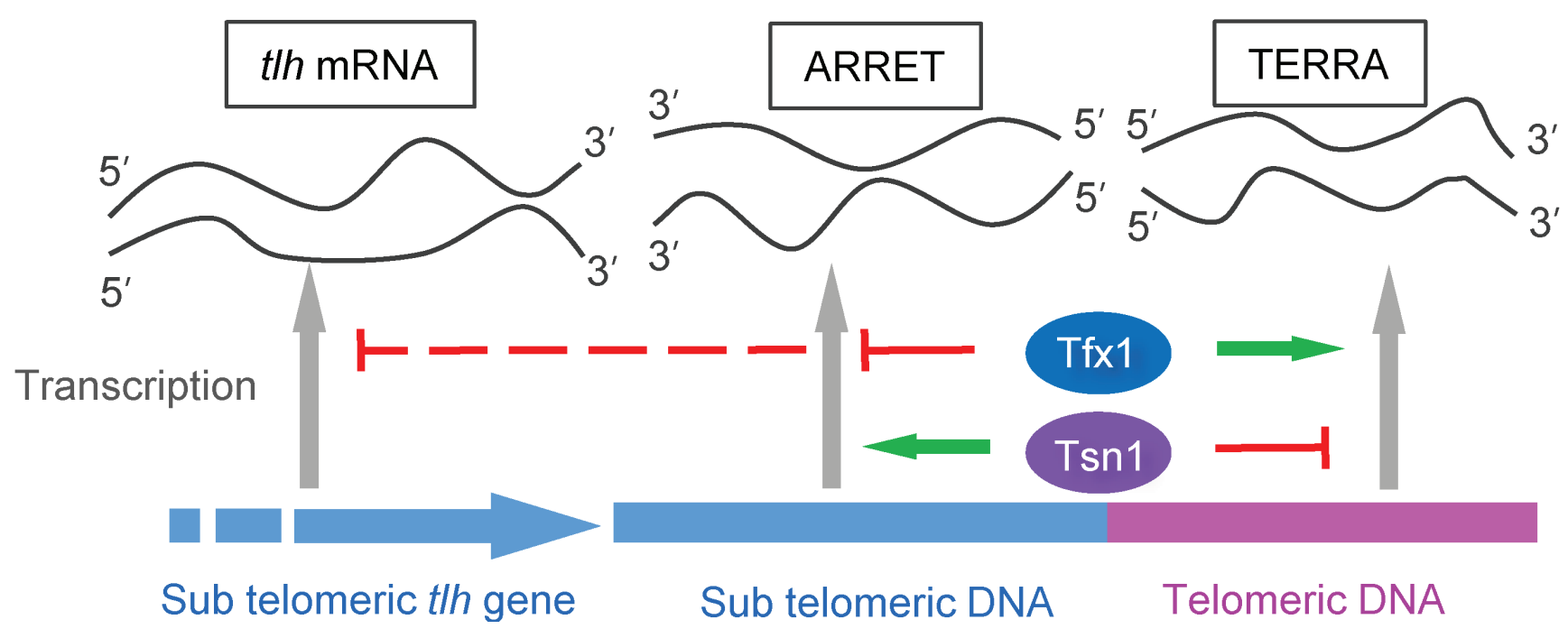

Figure 6: Model for the mechanism of telomere-associated transcript differential control by Tfx1 and Tsn1 in $S$. pombe. Tfx 1 serves to repress sub-telomeric ARRETs and $t$ th transcripts (upper red full/broken lines), but stabilizes (upper green arrow) elevated levels of TERRAs seen in the $t s n 1 \Delta$ mutant. Tsn1 plays a reciprocal role, normally suppressing TERRA levels (lower red line), but stabilizing (lower green arrow) the elevated ARRET levels (but not the elevated $t h$ transcript levels) seen in the $t f x 1 \Delta$ mutant. 
6). Previously, it has been demonstrated that mouse Trax can inhibit the binding of mouse Translin to mRNA [53], and a similar direct regulation might be occurring here to drive the inter paralogue modulation mechanism. Additionally, when $t s n 1^{+}$is mutated, Tfx1 levels become depleted [35], so the residual Tfx 1 found in the $t s n 1 \Delta$ mutant must be sufficient to maintain a functional role for telomere transcript level regulation.

Trax function has recently been linked to the DNA damage response [26] and TERRAs have been linked to the telomeric DNA damage response [12-16]. We previously found no altered sensitivity of the $S$. pombe tf $x 1 \Delta$ (or tsn $1 \Delta$ ) mutant to a wide range of DNA damaging agents [34], so there is no current evidence to link this phenomenon to telomeric DNA damage tolerance in $S$. pombe.

The finding that Translin and Trax control telomeric transcript homeostasis adds a new dimension to our understanding of these conserved nucleic acid regulators. It has recently been proposed that Translin and Trax may provide anti-cancer therapeutic targets [34]; however, before small molecule inhibitors are developed and applied to the clinical setting it is of fundamental importance that we fully elucidate the normal function of this protein pair to ensure that inhibition of their function does not adversely influence non-diseased tissues.

\section{MATERIALS AND METHODS}

\section{S. pombe strains and media}

A list of strains used in this study and their genotypes are shown in Table S1. Media, standard growth conditions, $S$. pombe transformations and genetic analyses were as described by Forsburg and Rhind [54]. All gene deletions in strains were created as described by Bähler and co-workers [55].

\section{S. pombe DNA extraction and Southern blotting}

DNA extraction was performed as described in Forsburg and Rhind [54]. After digestion with ApaI (New England Biolabs), $15 \mu \mathrm{g}$ of DNA was separated on a $1.2 \%$ agarose gel, transferred to positively charged nylon membrane (GeneScreen Plus) and hybridized to a radioactively labelled probe at $42^{\circ} \mathrm{C}$ for 18 hours in Church Gilbert buffer. Oligonucleotide probe corresponding to the telomeric G-strand was 5'-end labelled with T4 polynucleotide kinase (New England Biolabs) in the presence of $\left[\gamma-{ }^{32} \mathrm{P}\right] \mathrm{ATP}$. The probe sequence was 5'- GGGTTACAGGTTACAGGTTACA-3'. After hybridization, membranes were washed three times in $6 \mathrm{x}$ $\mathrm{SSC} / 0.1 \% \mathrm{SDS}$ at room temperature. Radioactive signals were detected using a Molecular Imager FX (Bio-Rad) and analysed using Quantity One (Bio-Rad). Migration ratio was calculated as the ratio of the distance migrated for the $2 \mathrm{~kb}$ marker as a fraction of the distance migrated by the telomere probe signal midpoint as determined by Quantity One. Mean values were obtained from four independently generated blots.

\section{Human cell cultures}

Human SW480 cell line was obtained from the European Collection of Cell Culture. They were grown in Invitrogen Dulbeco's Modified Eagle's medium (DMEM+Glutamax) supplemented with $10 \%$ foetal bovine serum (Life Technologies) in a $37^{\circ} \mathrm{C}$ incubator with $5 \% \mathrm{CO}_{2}$ SW480 cells were authenticated prior to use using LGC Standards Cell Line Authentication service (tracking number 710418378). Cells were checked for mycoplasma at regular intervals using the LookOut ${ }^{\circledR}$ Mycoplasma Detection Kit (Sigma Aldrich; MP0035).

\section{RNA extraction and reverse transcription PCR}

Total RNA was isolated from $S$. pombe cells using the MasterPure ${ }^{\mathrm{TM}}$ Yeast RNA Purification Kit (Epicentre) following the manufacturer's instructions and treated with RNase-free DNase (Promega). Total RNA was extracted from human SW480 cells using TRIzol (Life Technologies) as described by Feichtinger and co-workers [56]. $1 \mu \mathrm{g}$ of RNA was used as template in first strand synthesis by Superscript III (Invitrogen). The RNA was denatured in the presence or absence of primer at $90^{\circ} \mathrm{C}$ for 1 minute followed by cooling to $55^{\circ} \mathrm{C}$ before adding the reverse transcriptase mix and incubating for 50 minutes. A volume of $2 \mu \mathrm{l}$ of cDNA was PCR amplified using MyTaq $^{\text {TM }}$ Red Mix (Bioline). PCR with subtelomeric primers was carried out for 3 minutes at $95^{\circ} \mathrm{C}$, then 35 cycles of $95^{\circ} \mathrm{C}$ for 30 seconds, $62^{\circ} \mathrm{C}$ for 20 seconds, $72^{\circ} \mathrm{C}$ for 20 seconds, followed by $72^{\circ} \mathrm{C}$ for 5 minutes. The same cycling conditions were used for act1, except that an annealing temperature of $58^{\circ} \mathrm{C}$ was used for 25 cycles.

RNA reversed transcribed with Superscript III was also used for TERRA qRT-PCR experiments. cDNA was amplified using the GoTaq ${ }^{\circledR}$ qPCR Master Mix (Promega) on a CFX96 real-time system (Bio-Rad) following the manufacturer's protocol. Sequences of all primers used in the study are given in Table S2.

\section{Whole transcriptome microarray analysis}

Appropriate strains were cultured to an absorbance of 0.5 at $600 \mathrm{~nm}$ and RNA was collected from cells using the hot phenol method. The RNA was processed as described by Kowalik and co-workers [57] following the processed described in the GeneChip Whole Transcript 
Double-Stranded Target Assay Manual from Affymetrix using the GeneChip S. pombe Tiling 1.0FR. Data processing is described in the Supplemental methods.

\section{TSN and TSNAX depletion in SW480 cells}

SW480 cells were transfected with pre-designed siRNAs directed to TSNAX (Qiagen, SI00751982) and TSN (Qiagen,SI03650318) using HiPerFect Transfection Reagent (Qiagen). After three hits, each lasting 24 hours, RNA was extracted using RNeasy Mini Kit (Qiagen) and reverse transcribed using the SuperScript III First-Strand Synthesis System (Invitrogen) in the presence of oligo dT cDNA was amplified using the GoTaq ${ }^{\circledR}$ qPCR Master Mix (Promega) on a CFX96 real-time system (Bio-Rad) following the manufacturer's protocol. Primer sequences are provided in Table S2.

\section{ACKNOWLEDGMENTS}

The authors would like to thank C. Norbury for sharing unpublished information on the number of $S$. pombe th genes. We would also like to thank J. Cooper for $S$. pombe strains and M. Bühler for access to array facilities.

\section{CONFLICTS OF INTEREST}

There are no known conflicts of interest associated with this work.

\section{GRANT SUPPORT}

This work was funded in part by awards from Cancer Research Wales, the Government of the Kingdom of Saudi Arabia (to R.J.M. and J.A.W.) and the OMICS Center Graz grant of the Austrian Ministry for Science, Research and Economy (to G.G.T.).

\section{REFERENCES}

1. Santaguida S, Amon A. Short- and long-term effects of chromosome mis-segregation and aneuploidy. Nat Rev Mol Cell Biol. 2015; 16: 473-485.

2. Jain D, Cooper JP. Telomeric strategies: means to an end. Annu Rev Genet. 2010; 44: 243-269.

3. Hockemeyer D, Collins K. Control of telomerase action at human telomeres. Nat Struct Mol Biol. 2015; 22: 848-852.

4. Schmidt JC, Cech T. Human telomerase: biogenesis, trafficking, recruitment, and activation. Genes Dev. 2015; 9: 1095-1105.

5. Martinez P, Blasco MA. Replicating through telomeres: a means to an end. Trends Biochem Sci. 2015; 40: 504-515.

6. Pickett HA, Reddel RR. Molecular mechanisms of activity and derepression of alternative lengthening of telomeres. Nat Struct Mol Biol. 2015; 22: 875-880.

7. Novo CL, London̆o-Vallejo JA. Telomeres and the nucleus. Semin Cancer Biol. 2013; 23: 116-124.

8. Sarek G, Marzec P, Margalef P, Boulton SJ. Molecular basis of telomere dysfunction in human genetic diseases. Nat Struct Mol Biol. 2015; 2: 867-874.

9. de Lange T. Shelterin: the protein complex that shapes and safeguards human telomeres. Genes Dev. 2005; 19: 21002110 .

10. Bühler M, Gasser SM. Silent chromatin at the middle and ends: lessons from yeast. EMBO J. 2009; 28: 2149-2161.

11. Schoeftner S, Blasco MA. A 'higher order' of telomere regulation: telomere heterochromatin and telomeric RNAs. EMBO J. 2009; 28: 2323-2336.

12. Azzalin CM, Lingner J. Telomere functions grounding on TERRA firma. Trends Cell Biol. 2015; 25: 29-36.

13. Cusanelli E, Chartrand P. Telomeric repeat-containing RNA TERRA: a noncoding RNA connecting telomere biology to genome integrity. Front Genet. 2015; 6: 143.

14. Maicher A, Lockhart A, Luke B. Breaking new ground: digging into TERRA function. Biochim Biophys Acta. 2014; 1839: 387-394.

15. Rippe K, Luke B. TERRA and the state of the telomere. Nat Struct Mol Biol. 2015; 22: 853-858.

16. Wang $\mathrm{C}$, Zhao L, Lu S. Role of TERRA in the regulation of telomere length. Int J Biol Sci. 2015; 11: 316-323.

17. Aoki K, Suzuki K, Sugano T, Tasaka T, Nakahara K, Kuge O, Omori A, Kasai M. A novel gene, Translin, encodes a recombination hotspot binding protein associated with chromosomal translocations. Nat Genet. 1995; 10: 167-174.

18. Jaendling A, McFarlane RJ. Biological roles of translin and translin-associated factor-X: RNA metabolism comes to the fore. Biochem J. 2010; 429: 225-234.

19. Wu XQ, Gu W, Meng X, Hecht NB. The RNA-binding protein, TB-RBP, is the mouse homologue of translin, a recombination protein associated with chromosomal translocations. Proc Natl Acad Sci USA. 1997; 94: 56405645.

20. Parizotto EA, Lowe ED, Parker JS. Structural basis for duplex RNA recognition and cleavage by Archaeoglobus fulgidus C3PO. Nat Struct Mol Biol. 2013; 20: 380-386.

21. Eliahoo E, Ben Yousef R, Pérez-Cano L, Fernández-Recio J, Glaser F, Manor H. Mapping the interaction sites of the Schizosaccharomyces pombe protein Tranlisn with nucleic acids and proteins: a combined molecular genetics and bioinformatics study. Nucleic Acids Res. 2010; 38: 29752989.

22. Li L, Gu W, Liang C, Liu Q, Mello CC, Liu Y. The translin-TRAX complex (C3PO) is a ribonuclease in tRNA processing. Nat Struct Mol Biol. 2012; 19: 824-830.

23. Wang J, Boja ES, Oubrahim H, Chock PB. Testis brain ribonucleic acid-binding protein/translin possesses both 
single-stranded and double-stranded ribonuclease activities. Biochemistry. 2004; 43: 13424-13431.

24. Jacob E, Pucshansky L, Zeruya E, Baran N, Manor H. The human protein translin specifically binds single-stranded microsatellite repeats, $\mathrm{d}(\mathrm{GT}) \mathrm{n}$, and G-strand telomeric repeats, d(TTAGGG)n: a study of the binding parameters. J Mol Biol. 2004; 344: 939-950.

25. Arnoult N, Karlseder J. Complex interaction between the DNA-damage response and mammalian telomeres. Nat Struct Mol Biol. 2015; 22: 859-866.

26. Wang JY, Chen SY, Sun CN, Chien T, Chern Y. A central role of TRAX in the ATM-mediated DNA repair. Oncogene. 2015; doi: 10.1038/onc.2015.228.

27. Fukuda Y, Ishida R, Aoki K, Nakahara K, Takashi T, Mochida, Suzuki O, Matsuda J, Kasai M. Contribution of Translin to hematopoietic regeneration after sublethal ionizing irradiation. Biol Pharm Bull. 2008; 31: 207-211.

28. Li Z, Wu Y, Baraban JM. The Translin/Trax RNA binding complex: clues to function in the nervous system. Biochim Biophys Acta. 2008; 1779: 479-485.

29. Chiaruttini C, Vicario A, Li Z, Baj G, Braiuca P, Wu Y, Lee FS, Gardossi L, Baraban JM, Tongiorgi E. Dendritic trafficking of BDNF mRNA is mediated by translin and blocked by the G196A (Val66Met) mutation. Proc Natl Acad Sci USA. 2009; 106: 16481-16486.

30. Liu Y, Ye X, Jiang F, Laing F, Chen D, Peng J, Kinch LN, Grishin NV, Liu Q. C3PO, an endoribonuclease that promotes RNAi by facilitating RISC activation. Science. 2009; 325: 750-753.

31. Tian Y, Simanshu DK, Ascano M, Diaz-Avalos R, Park AY, Juranek SA, Rice WJ, Yin Q, Robinson CV, Tuschi T, Patel DJ. Multimeric assembly and biochemical characterization of the Trax-Translin endonuclease complex. Nat Struct Mol Biol. 2011; 18: 658-664.

32. Ye X, Huang N, Liu Y, Paroo Z, Huerta C, Li P, Chen S, Liu Q, Zhang H. Structure of C3PO and mechanism of human RISC activation. Nat Struct Mol Biol. 2011; 18: 650-657.

33. Holoch D, Moazed D. RNA-mediated epigenetic regulation of gene expression. Nat Rev Genet. 2015; 16: 71-84.

34. Asada K, Canestrari E, Fu X, Li Z, Makowski E, Wu YC, Mito JK, Kirsch DG, Baraban J, Paroo Z. Rescuing dicer defects via inhibition of an anti-dicing nuclease. Cell Rep. 2014; 9: 1471-1481.

35. Jaendling A, Ramayah S, Pryce DW, McFarlane RJ. Functional characterisation of the Schizosaccharomyces pombe homologue of the leukaemia-associated translocation breakpoint binding protein translin and its binding partner, TRAX. Biochim Biophys Acta. 2008; 1783: 203-213.

36. Laufman O, Ben Yosef R, Adir N, Manor H. Cloning and characterization of the Schizosaccharomyces pombe homologs of the human protein Translin and Translinassociated protein TRAX. Nucleic Acids Res. 2005; 33: 4128-4139.
37. Mandell JG, Goodrich KJ, Bähler J, Cech TR. Expression of a RecQ helicase homolog affects progression through crisis in fission yeast lacking telomerase. J Biol Chem. 2005; 280: 5249-5257.

38. Cooper JP, Nimmo ER, Allshire RC, Cech TR. Regulation of telomere length and function by a Myb-domain protein in fission yeast. Nature. 1997; 385: 744-747.

39. Hansen KR, Ibarra PT, Thon G. Evolutionary-conserved telomere-linked helicase genes of fission yeast are repressed by silencing factors, RNAi components and the telomerebinding protein Taz1. Nucleic Acids Res. 2006; 34: 78-88.

40. Tadeo X, Wang J, Kallgren SP, Liu J, Reddy BD, Qiao F, Jia S. Elimination of shelterin components bypasses RNAi for pericentric heterochromatin assembly. Genes Dev. 2013; 27: 2489-2499.

41. Bah A, Wischnewski H, Shchepachev V, Azzalin CM. The telomeric transcriptome of Schizosaccharomyces pombe. Nucleic Acids Res. 2012; 40: 2995-3005.

42. Greenwood J, Cooper JP. Non-coding telomeric and subtelomeric transcripts are differentially regulated by telomeric and heterochromatin assembly factors in fission yeast. Nucleic Acids Res. 2012; 40: 2956-2963.

43. Lorenzi LE, Bah A, Wischnewski H, Shchepachev V, Soneson C, Santagostino M, Azzalin CM. Fission yeast Cactin restricts telomere transcription and elongation by controlling Rap1 levels. EMBO J. 2015; 34: 115-129.

44. Schoeftner S, Blasco MA. Developmentally regulated transcription of mammalian telomeres by DNA-dependent RNA polymerase II. Nat Cell Biol. 2008; 10: 228-236.

45. Azzalin CM, Reichenbach P, Khoriauli L, Giulotto E, Lingner J. Telomeric repeat containing RNA and RNA surveillance factors at mammalian chromosome ends. Science. 2007; 318: 798-801.

46. Porro A, Feuerhahn S, Reichenbach P, Lingner J. Molecular dissection on telomeric repeat-containing RNA biogenesis unveils the presence of distinct and multiple regulatory pathways. Mol Cell Biol. 2010; 30: 4808-4817.

47. Yang S, Hecht NB. Translin associated protein $\mathrm{X}$ is essential for cellular proliferation. FEBS Lett. 2004; 576: 221-225.

48. Yang S, Cho YS, Chennathukuzhi VM, Underkoffler LA, Loomes K, Hecht NB. Tranlsin-associated factor X is posttranscriptionally regulated by its partner protein TB-RBP, and both are essential for normal cell proliferation. J Biol Chem. 2004; 279: 12605-12614.

49. Ishida R, Okado H, Sato H, Shionoiri C, Aoki K, Kasai M. A role for the octomeric ring protein, Translin, in mitotic cell division. FEBS Lett. 2002; 525: 105-110.

50. Luke B, Panza A, Redon S, Iglesias N, Li Z, Lingner J. The Ratlp 5' to 3' exonuclease degrades telomeric repeatcontaining RNA and promotes telomere elongation in Saccharomyces cerevisiae. Mol Cell. 2008; 32: 465-477.

51. Luo Z, Dai Z, Xie X, Feng X, Liu D, Songyang Z, Xiong Y. TeloPIN: a database of telomeric proteins interaction 
network in mammalian cells. Database. 2015; 2015:pii: bav018.

52. Eliahoo E, Litovco P, Ben Yosef R, Bendalak K, Ziv T, Manor H. Identification of proteins that form specific complexes with the highly conserved protein Translin in Schizosaccharomyces pombe. Biochim Biophys Acta. 2014; 1844: 767-777.

53. Chennsthukuzhi V, Kurihara Y, Bray JD, Hecht NB. Trax (translin-associated factor $\mathrm{X}$ ), a primarily cytoplasmic protein, inhibits the binding of TB-RBP (translin) to RNA. J Biol Chem. 2001; 276: 13256-13263.

54. Forsburg SL, Rhind N. Basic methods for fission yeast. Yeast 2006; 23: 173-183.

55. Bähler J, Wu JQ, Longtine MS, Shah NG, McKenzie A 3rd, Steever AB, Wach A, Philippsen P, Pringle JR. Heterologous modules for efficient and versatile PCR-based gene targeting in Schizosaccharomyces pombe. Yeast. 1998; 14: 943-951.
56. Feichtinger J, Aldeailej I, Anderson R, Almutairi M, Almatrafi A, Alsiwiehri N, Griffiths K, Stuart N, Wakeman JA, Larcombe L, McFarlane RJ. Meta-analysis of clinical data using human meiotic genes identifies a novel cohort of highly restricted cancer-specific marker genes. Oncotarget. 2012; 3: 843-853. doi: 10.18632/oncotarget.580.

57. Kowalik KM, Shimada Y, Flury V, Stadler MB, Batki J, Bühler M. The Pafl complex represses small-RNAmediated epigenetic gene silencing. Nature. 2015; 520: 248-252. 